\title{
A case of bicuspid aortic valve with two raphes
}

\author{
Go Hashimoto $\cdot$ Makoto Suzuki $\cdot$ Hisao Yoshikawa \\ Takenori Otsuka $\cdot$ Yukiko Kusunose • \\ Masato Nakamura $\cdot$ Kaoru Sugi
}

Received: 24 August 2012/Revised: 16 January 2013/Accepted: 18 January 2013/Published online: 9 February 2013

(C) The Author(s) 2013. This article is published with open access at Springerlink.com

Case

A 60-year-old woman was referred to our hospital for exertional shortness of breath. She was made aware of a heart murmur as a high school student. She had exertional dyspnea and chest pain. Her symptoms were getting worse over the previous year. On admission, her blood pressure was $160 / 54 \mathrm{mmHg}$, heart rate was $72 \mathrm{bpm}$, and grade 3/6 regurgitant murmur over the precordium was present. She was diagnosed with severe aortic regurgitation by transthoracic echocardiography and the other clinical examination.

Transthoracic echocardiography showed severe aortic regurgitation with normal left ventricular systolic function. Two raphes of the aortic valve were not diagnosed by transthoracic echocardiography because of poor quality echo images. Two-dimensional transesophageal echocardiograms showed a bicuspid aortic valve (BAV) with fused cusps between the two components of the right and noncoronary cusps (Fig. 1). Furthermore there were two raphes on the fused cusps. Real-time three-dimensional echocardiograms showed two commissures and two raphes on the fused area in systole and diastole (Fig. 2a, b). The perioperative findings were exactly the same as the information obtained by three-dimensional transesophageal echocardiography (Fig. 2c).

G. Hashimoto $(\bowtie) \cdot$ M. Suzuki $\cdot$ H. Yoshikawa $\cdot$ T. Otsuka · Y. Kusunose $\cdot$ M. Nakamura $\cdot$ K. Sugi

Division of Cardiovascular Medicine, Toho University Ohashi Medical Center, 2-17-6, Ohashi, Meguro-ku, Tokyo, Japan e-mail: golegguards@yahoo.co.jp

\section{Discussion}

The bicuspid aortic valve is a common congenital cardiac anomaly, having an incidence in the general population of 0.9-2.0\% [1]. The term "BAV" includes different morphologic phenotypes [2, 3]. The "purely" BAV is composed of two cusps, morphologically and functionally. However, the most frequent form of BAV consists of three developmental anlagen of cusps with one raphe and two commissures. This case has a rare morphology with two raphes and two commissures. Transesophageal echocardiography demonstrates two raphes clearly on the fused area of both the right and non-coronary cusps. The threedimensional transesophageal echocardiographic images help provide more detailed structural and functional features of the aortic valve. Sievers and Schmidtke [3] reported the three characteristics required for $\mathrm{BAV}$

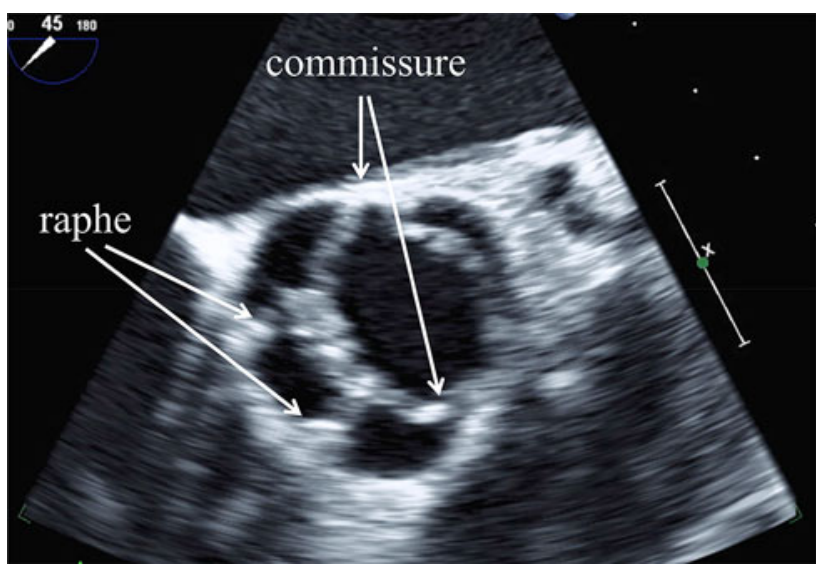

Fig. 1 Two-dimensional transesophageal echocardiographic image of the aortic valve. Two raphes and two commissures were clearly demonstrated in systole 

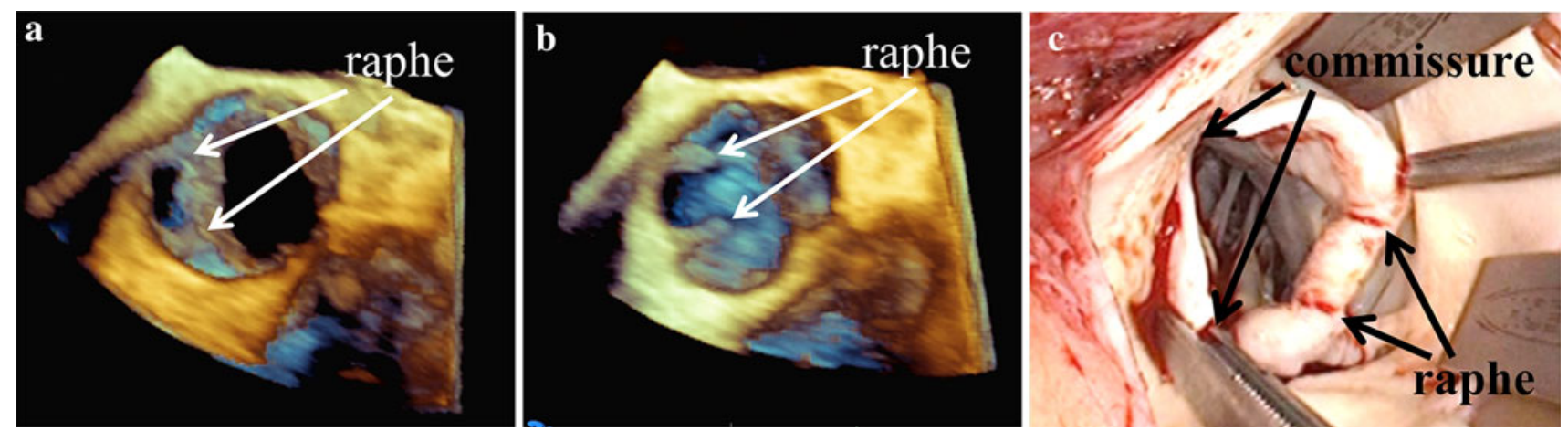

Fig. 2 Real-time three-dimensional echocardiograms showed two commissures and two raphes on the fused area of both the right and non-coronary cusps in systole (a) and diastole (b). A photograph of

classification: number of raphes, spatial position of cusps or raphes, and functional status of the valve. However, our case does not belong to any classification in their report. Jasper et al. [4] reported a case of bicuspid aortic valve with a double raphe. Their case had a bicuspid aortic valve with two separate raphes in each cusp. Our case has double raphes in one fused cusp. Transesophageal echocardiography provided comprehensive images in this case which has two raphes and two commissures.

Conflict of interest The authors have no conflict of interest in connection with this paper.

Open Access This article is distributed under the terms of the Creative Commons Attribution License which permits any use, distribution, and reproduction in any medium, provided the original author(s) and the source are credited. the actual operation view shows two commissures and two raphes on the fused area of both the right and non coronary cusps (c)

\section{References}

1. Ward C. Clinical significance of the bicuspid aortic valve. Heart. 2000;83:81-5.

2. Sabet HY, Edwards WD, Tazelaar HD, et al. Congenitally bicuspid aortic valves: a surgical pathology study of 542 cases (1991 through 1996) and a literature review of 2,715 additional cases. Mayo Clin Proc. 1999;74:14-26.

3. Sievers H-H, Schmidtke C. A classification system for the bicuspid aortic valve from 304 surgical specimens. J Thorac Cardiovasc Surg. 2007;133(5):1226-33.

4. Jasper J, Kurvers M, Kofflard MJM. Bicuspid aortic valve with vertical opening and double raphe. Eur Heart J Cardiovasc Imaging. 2012;13:123. 